\title{
Oral health may prevent severe COVID-19 disease
}

The potential for healthy gums to reduce the severity of COVID-19 disease is outlined in a paper recently published in the Journal of Oral Medicine and Dental Research. ${ }^{1}$

The paper follows evidence published earlier this year in the Journal of Clinical Periodontology, the official publication of the European Federation of Periodontology (EFP), that patients with COVID-19 were three times more likely to experience complications if they also had gum disease. $^{2}$

The authors compiled existing evidence to propose a pathway by which the severe acute respiratory syndrome coronavirus 2 (SARS-CoV-2) is transmitted to the lungs, where it causes COVID-19 lung disease.

They suggest that the virus enters the body through the upper airways (nose and mouth), collects in the saliva in the mouth and enters dental plaque under the gums. It then crosses the gums into the blood vessels, where it travels to the arteries in the lungs - rather than travelling to the lungs via the airways. The biological basis for this route of infection is outlined. In addition, the authors put forward the idea that diseased or damaged gums could weaken the mucosal barrier in the mouth and allow the virus to more easily enter the bloodstream.

According to the paper: 'If confirmed, this hypothetical model may provide a rationale for understanding why some individuals develop COVID-19 lung disease and others do not. It would also fundamentally change the way COVID-19 is managed, providing a new line of exploration into treatments targeted at the source of the viral reservoir, the mouth'. If correct, 'simple antimicrobial oral healthcare measures could be implemented not only with the aim of reducing the risk of transmission between individuals' but also as 'a means of mitigating the risk of developing lung disease, and therefore the most severe form of the disease'.

Co-author Professor Iain Chapple of the University of Birmingham said: 'Dental plaque contains billions of microbes and needs to be removed daily. Otherwise, the gums become inflamed and small ulcers that communicate directly with the bloodstream form between the gums and teeth. These allow microbes like viruses to enter the bloodstream. The plaque under the gums forms a biofilm, which acts as a self-protective environment for many microbes to survive and prosper. Given the high SARS-CoV-2 viral load in saliva, this bloodborne route of transfer to the lungs is highly plausible'.

Professor Nicola West, EFP secretary general, said: 'More research is needed to substantiate this theory, but in the meantime, it seems sensible to take extra care in looking after our teeth and gums, get regular check-ups and undergo treatment when needed'.

The EFP has recently edited the short animated film 'Gum disease and Covid-19 complications': https://bit.ly/3mx8TRK.

\section{References}

1. Lloyd-Jones G, Molayem S, Pontes C C, Chapple I. The COVID-19 pathway: A proposed oralvascular-pulmonary route of SARS-CoV-2 infection and the importance of oral healthcare measures. J Oral Med Dent Res 2021; 2(1)-S1. Available at: https://www.genesispub.org/j-oralmed-and-dent-res/the-COVID-19-pathway-a-proposed-oral-vascular-pulmonary-route-ofsars-cov-2-infection-and-the-importance-of-oral-healthcare-measures (accessed April 2021). 2. Marouf N, Cai W, Said K N et al. Association between periodontitis and severity of COVID19 infection: a case-control study. J Clin Periodontol 2021. doi:10.1111/jcpe.13435. 